\title{
INFLUENCE OF THE INTERMEDIATE MATERIAL ON THE SINGULAR STRESS FIELD IN TRI-MATERIAL JUNCTIONS
}

\author{
A. CARPINTERI AND M. PAGGI \\ Department of Structural and Geotechnical Engineering, Politecnico di Torino, \\ C.so Duca degli Abruzzi 24, 10129 Torino, Italy
}

\begin{abstract}
According to the mathematical formalism of the eigenfunction expansion method, the problem of stresssingularities arising from multi-material junctions is addressed. The wedges are composed of isotropic homogeneous materials and are in a condition of plane stress or strain. The order of the stress-singularity is provided for tri-material junctions, paying special attention to the role played by Mode-I and Mode-II deformation. The effect of cracks inside either the softer or the stiffer material is also investigated. Numerical results can be profitably used for establishing optimum material configurations.
\end{abstract}

\section{INTRODUCTION}

According to Linear Elastic Fracture Mechanics, stress-singularities occur in multi-material junctions. Such problems have been extensively studied in composite plates since the pioneering papers by Williams [1,2], Bogy [3,4] and Theocaris [5].

The aim of the present paper is to investigate, from the theoretical point of view, on the order of the stress-singularity arising from tri-material junctions. These boundary-value problems in plane elasticity have been mainly solved by using either the Mellin transform technique [6,7] or the Muskhelishvili complex function representation $[5,8,9]$. In the present paper, the general problem of multi-material junctions is formulated within the framework of the eigenfunction expansion method [1]. Numerical examples concerning several tri-material configurations will show the effectiveness of the approach. On the basis of the proposed method, the order of the stress-singularity for different material combinations is provided. According to the criterion of minimum singularity [9], optimum configurations can be determined. Eventually, the influence of initial defects is taken into account by considering the limit cases of cracks inside either the softer or the stiffer material.

\section{MATHEMATICAL FORMULATION}

The geometry of a plane problem consisting of $n$ dissimilar wedges of arbitrary angles perfectly bonded along their interfaces which converge at the same vertex $O$ is depicted in Fig. 1a. Each of the material regions is denoted by $\Omega_{i}$ with $i=0 \ldots n-1$, and it is comprised between the interfaces $\Gamma_{i}$ and $\Gamma_{i+1}$. The first and the last interfaces, corresponding respectively to $\theta=0$ and $\theta=2 \pi$, coincide and are referred to as $\Gamma_{0}$.

According to Williams [1], it is possible to assume, for the $i$-th subregion, the following separable form for the bi-harmonic stress function $\Phi_{i}$ :

$$
\Phi_{i}(r, \theta)=\sum_{j} r^{\lambda} f_{i, j}\left(\theta, \lambda_{j}\right),
$$

where $\lambda_{j}$ and $f_{i, j}$ are referred to as eigenvalues and eigenfunctions, respectively. The summation with respect to $j$ is introduced in eqn (1), since it is possible to have more than one eigenvalue for each problem. The bi-harmonic condition requires $f_{i}$ to be of the form: 


$$
f_{i}(\theta, \lambda)=A_{i} \sin (\lambda+1) \theta+B_{i} \cos (\lambda+1) \theta+C_{i} \sin (\lambda-1) \theta+D_{i} \cos (\lambda-1) \theta,
$$

where $\mathrm{A}_{i}, \mathrm{~B}_{i}, \mathrm{C}_{i}$ and $\mathrm{D}_{i}$ are undetermined constants. For each eigenvalue $\lambda$ the corresponding stress and displacement fields are given by:

$$
\begin{array}{rlrl}
\sigma_{r}^{i} & =r^{\lambda-1}\left[f_{i}^{\prime \prime}+(\lambda+1) f_{i}\right] & u^{i}{ }_{r}=\frac{r^{\lambda}}{2 G_{i}}\left\{-(\lambda+1) f_{i}+\frac{1}{\lambda \kappa_{i}}\left[f_{i}^{\prime \prime}+(\lambda+1)^{2} f_{i}\right]\right\} \\
\sigma_{\theta}^{i}=r^{\lambda-1}\left[\lambda(\lambda+1) f_{i}\right] & u^{i} \theta=\frac{r^{\lambda}}{2 G_{i}}\left\{-f_{i}^{\prime}-\frac{1}{\lambda(\lambda-1) \kappa_{i}}\left[f_{i}^{\prime \prime \prime}+(\lambda+1)^{2} f_{i}^{\prime}\right]\right\} \\
\tau_{r \theta}^{i}=r^{\lambda-1}\left[-\lambda f_{i}^{\prime}\right] &
\end{array}
$$

where primes denote differentiation with respect to $\theta$. In the above equations, $\mathrm{G}_{i}$ is the shear modulus and $\kappa_{i}$, in terms of the Poisson's ratio, is given by either $1+v_{i}$ for plane stress or $1 /\left(1-v_{i}\right)$ for plane strain.
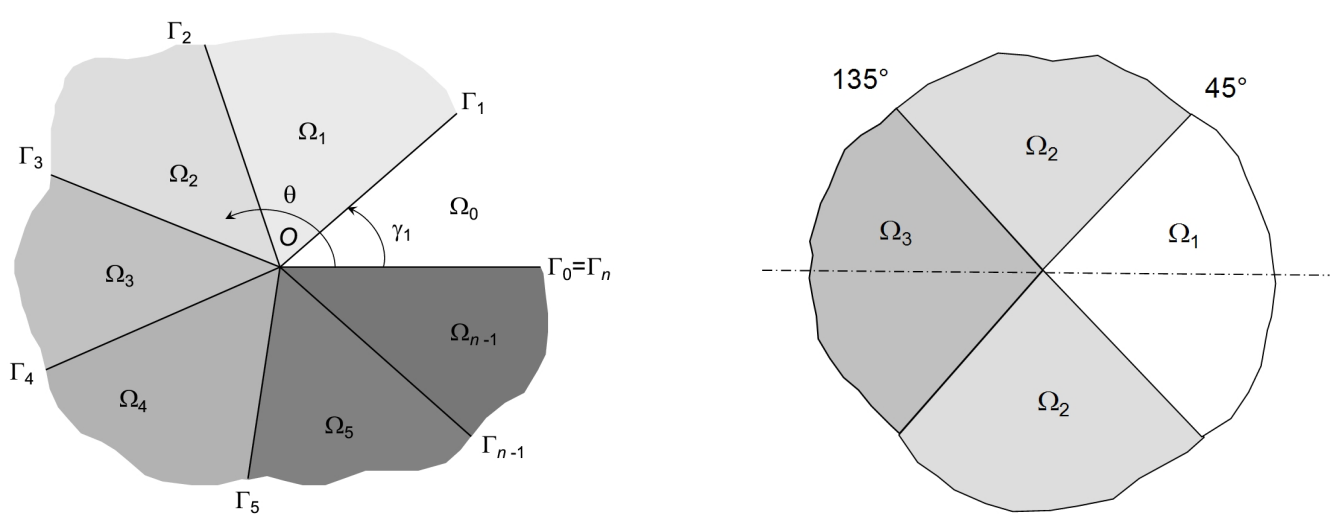

(a)

(b)

Figure 1: Scheme of (a) a general multi-material problem and of (b) a tri-material junction configuration investigated in the present paper.

The following boundary conditions at the interface $\Gamma_{\mathrm{i}}$ can be written in the case of perfect bonding:

$$
\begin{array}{ll}
\sigma_{\theta}^{i}\left(r, \gamma_{i+1}\right)=\sigma_{\theta}^{i+1}\left(r, \gamma_{i+1}\right), & u_{r}^{i}\left(r, \gamma_{i+1}\right)=u_{r}^{i+1}\left(r, \gamma_{i+1}\right), \\
\tau_{r \theta}{ }^{i}\left(r, \gamma_{i+1}\right)=\tau_{r \theta}{ }^{i+1}\left(r, \gamma_{i+1}\right), & u_{\theta}^{i}\left(r, \gamma_{i+1}\right)=u_{\theta}^{i+1}\left(r, \gamma_{i+1}\right),
\end{array}
$$

paying attention to the fact that the interface $\Gamma_{0}$ must be defined by $\gamma=0$ for region 1 , and $\gamma_{n+1}=2 \pi$ for region $n$. This method yields to a set of $4 n$ equations in $4 n+1$ unknowns $\mathrm{A}_{i}, \mathrm{~B}_{i}, \mathrm{C}_{i}, \mathrm{D}_{i}$, and $\lambda$, which can be symbolically written as:

$$
\Lambda \mathbf{v}=\mathbf{0},
$$

where $\Lambda$ denotes the coefficient matrix which depends on $\lambda$, and $\mathbf{v}$ represents the vector which collects the unknowns $\mathrm{A}_{i}, \mathrm{~B}_{i}, \mathrm{C}_{i}, \mathrm{D}_{i}$. A nontrivial solution to the equation system (5) exists only if 
the determinant of the coefficient matrix vanishes. This yields to a characteristic equation which has to be solved for eigenvalues $\lambda$ which are in general complex. According to the present purposes, we are concerned only with those values of $\lambda$ which may lead to singularities in the stress field. This fact, together with the condition of continuity of the displacement field at the vertex where regions meet, imply that we are seeking for eigenvalues in the range $0<\operatorname{Re} \lambda<1$. Then, to find solutions to the eigenequations, a numerical iterative technique is employed.

Furthermore, as argued by Chen and Nisitani [8], when there exists a geometric symmetry in the problem, it is possible to subdivide the elastic field into a symmetric part and a skewsymmetric part, namely into a part due to the Mode-I deformation, and a part due to the Mode-II deformation. In the former case, the following symmetric conditions are applied at $\theta=0$, $\pi$, instead of eqn (4):

$$
\begin{gathered}
\tau_{r \theta}{ }^{i}\left(r, \gamma_{i+1}\right)=0, \\
u_{\theta}{ }^{i}\left(r, \gamma_{i+1}\right)=0 .
\end{gathered}
$$

In the latter, skew-symmetric conditions are imposed:

$$
\begin{gathered}
\sigma_{\theta}{ }^{i}\left(r, \gamma_{i+1}\right)=0, \\
u_{r}^{i}\left(r, \gamma_{i+1}\right)=0 .
\end{gathered}
$$

In this way, the complete eigenequation of the problem is reduced to two factors: the former determines the eigenvalues corresponding to Mode-I deformation, whereas the latter determines the eigenvalues corresponding to Mode-II deformation.

\section{PERFECTLY BONDED TRI-MATERIAL JUNCTIONS}

A tri-material junction problem schematically depicted in Fig. $1 \mathrm{~b}$ is investigated. Two materials occupying two quarter planes with a specified ratio between their elastic moduli, $E_{3} / E_{1}$, are joined by an intermediate material whose elastic modulus, $E_{2}$, is varied from $E_{1}$ to $E_{3}$. Different mechanical configurations characterized by the ratio $E_{3} / E_{1}$ are considered. Real and imaginary parts of the eigenvalues are numerically computed and depicted in Fig. 2 as functions of the modular ratio $E_{2} / E_{1}$. The limit case given by $E_{3} / E_{1}=1$ corresponds to a bi-material junction whose eigenvalues are depicted in Fig. 2a. On the other hand, solutions to tri-material junctions characterized by $E_{3} / E_{1}=100$ and $E_{3} / E_{1}=10000$ are depicted in Fig. 2b,c, respectively. For such problems, the limit cases given by either $E_{2} / E_{1}=1$ or $E_{2} / E_{1}=E_{3} / E_{1}$ correspond to bi-material junctions whose eigenvalues were provided by Carpinteri and Paggi [10].

Thanks to the geometric symmetry of these problems, the roots of the eigenequations corresponding to either Mode-I or Mode-II deformation can be separately computed. It is interesting to notice that, regardless of the value of the elastic modular ratio $E_{3} / E_{1}$, there are some mechanical configurations admitting only one root of the eigenequation (see Fig. 2b,c). To be more specific, when the elastic modulus of the intermediate material is close to that of the softer component, no stress-singularities due to Mode-I deformation are found. On the other hand, when the Young's modulus of the intermediate material approaches that of the third material, Mode-II stress-singularities are avoided. These trends are completely general for this type of tri-material junction and can be observed for any given value of the parameter $E_{3} / E_{1}$ different from 1 .

In the case of Mixed Mode deformation, the minimum singularity criterion would suggest that the more suitable material configuration is attained for the case corresponding to the minimum eigenvalues. As a consequence, for such tri-material junction problems the optimum configuration 
would correspond to a stiffness ratio such that $\operatorname{Re} \lambda^{\mathrm{I}}=\operatorname{Re} \lambda^{\mathrm{II}}$ (see Fig. 2b,c). By considering trimaterial junctions with different values of the ratio $E_{3} / E_{1}$, the value of $E_{2} / E_{1}$ is computed according to the above criterion and depicted in Fig. $2 d$ vs. the ratio between the elastic moduli of materials 1 and 3 . From these results, the following regression curve is obtained:

$$
E_{2} / E_{1}=0.933\left(E_{3} / E_{1}\right)^{0.467} \cong \sqrt{E_{3} / E_{1}} .
$$

In practice, this condition rules out intermediate materials whose elastic moduli are close to that of the stiffer material. Furthermore, it is important to notice that all the roots are necessary to describe the stress state in both materials, since it cannot be concluded that any root is somehow less dominant.
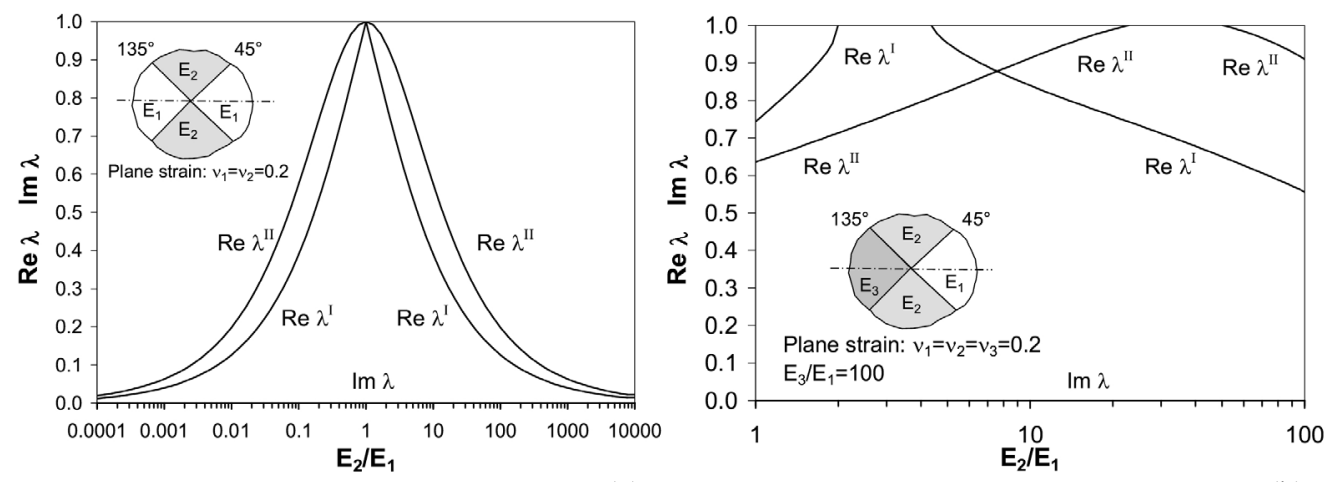

(a)

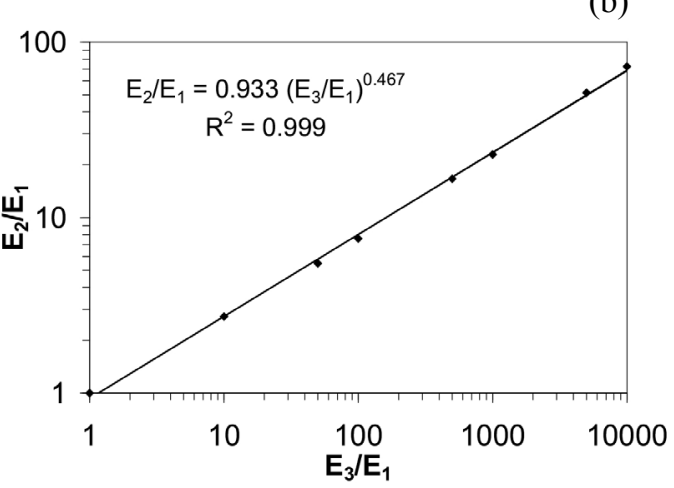

(c)

(d)

Figure 2: Eigenvalues for perfectly bonded tri-material junctions characterized by (a) $E_{3} / E_{1}=1$; (b) $E_{3} / E_{1}=100$; and (c) $E_{3} / E_{1}=10000$. (d) Relation between $E_{2} / E_{1}$ and $E_{3} / E_{1}$ according to the minimum singularity criterion.

\section{CRACKED TRI-MATERIAL JUNCTIONS}

Debonding along interfaces in tri-material junctions have been considered by Pageau et al. [9], whereas a little attention was paid to the problem of transgranular cracks. Hence, some of the previous results obtained for the order of the stress-singularity are extended to the case of a crack placed along the symmetry line inside material 1 or 3 . 
Considering as a representative case the a tri-material junction characterized by the ratio $E_{3} / E_{1}=100$ as that of the uncracked problem analyzed in Fig. 2b, the Young's modulus of the intermediate material is the independent variable of the problem and it is varied from $E_{1}$ to $E_{3}$. A crack is then considered either in the softer (Fig. 3a) or in the stiffer material (Fig. 3b). For each case study, real and imaginary parts of Mode-I and Mode-II eigenvalues are computed.

As a limit case, when the crack lies in the softer material and $E_{2} / E_{1}=1$, the problem reduces to a cracked bi-material junction with $E_{2} / E_{1}=1 / 100$. Analogously, when the tri-material junction is characterized by $E_{2} / E_{1}=100$, another bi-material problem is addressed. The same reasoning holds when the crack lies in the stiffer material and the limit cases of $E_{2} / E_{1}=1$ and $E_{2} / E_{1}=100$ are considered. Asymptotic results concerning such bi-material problems are provided in [10]. For a direct comparison between cracked and uncracked solutions, eigenvalues for the corresponding uncracked problems are depicted with dashed lines in Fig. 3.
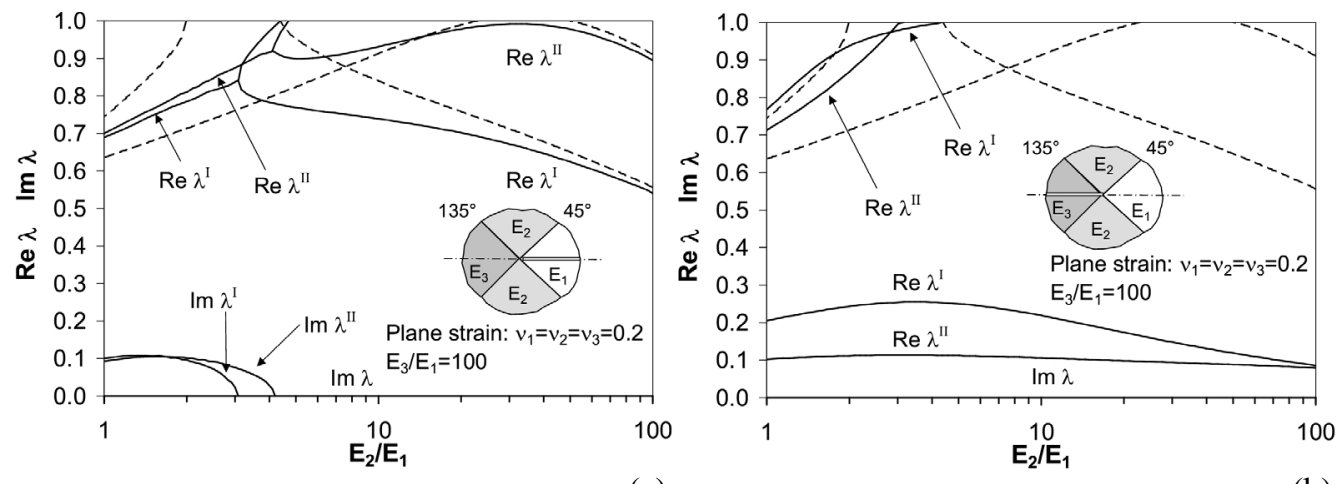

(a)

Figure 3: Eigenvalues for cracked tri-material junctions characterized by $E_{3} / E_{1}=100$. A crack lies either (a) in the softer or (b) in the stiffer material region.

When the crack lies in the softer material (see Fig. 3a), Mode-I and Mode-II eigenvalues remain greater than 0.5 , as in the uncracked problem. The main difference is represented by the fact that in the range $3<E_{2} / E_{1}<4$ Mode-II eigenvalues are complex and two real roots are found for Mode-I deformation. In this range Mode-II eigenvalues are complex. Furthermore, for $E_{2} / E_{1}$ approximately equal to 3 , a transition from two real roots to a complex conjugate pair also occurs for Mode-I eigenvalues.

On the other hand, a completely different behavior has to be noticed for a crack in the stiffer material (see Fig. 3b). Computed eigenvalues for this problem are real and two additional roots are found in the range $1<E_{2} / E_{1}<4$ with respect to the uncracked configurations. Such roots are far lower than 0.5 , i.e. the corresponding order of the stress-singularity is more severe than that due to a crack inside a homogeneous material. For $E_{2} / E_{1}>4$, the eigenvalues greater than 0.5 disappear and the stress state is characterized by the lower roots only.

As also observed for cracked bi-material junctions [10], these results imply that the asymptotic behavior of the singular stress field can be strongly influenced by cracks in the stiffer material. Both Mode-I and Mode-II stress-singularities are equally important for the description of the stress state in the tri-material regions. 


\section{CONCLUSIONS}

In the present paper the formulation of the general problem of multi-material junctions is provided according to the mathematical formalism of the eigenfunction expansion method. Numerical results concerning tri-material junctions perfectly bonded along their interfaces are obtained. Materials are linear elastic, isotropic and are in a condition of plane stress or strain. Thanks to the geometric symmetry of the considered problems, singularities due to either Mode-I or Mode-II deformation are separated. For some of the above geometries, the influence of transgranular cracks on the asymptotic state of stress was investigated.

From the engineering point of view, the obtained results constitute useful information about the variation of the order of the stress-singularity when the stiffness of one material varies with respect to the others. Contrarily to the results obtained by Pageau et al. [9] suggesting that an intermediate material increases the order of the stress-singularity in tri-material junction geometries when one material occupies a half plane region, we have shown that a third material can be favorable for the tri-material junctions herein investigated. The criterion of minimum singularity suggests that the best choice is given by an intermediate material whose elastic modulus is closer to that of the softer component.

Eventually, results concerning cracked geometries demonstrate that tri-material junctions are significantly influenced by cracks in the stiffer material. Numerical results show that all the eigenvalues must be taken into account for an accurate elastic stress analysis.

\section{ACKNOWLEDGEMENTS}

Support by the Italian Ministry of University and Scientific Research (MIUR) is gratefully acknowledged.

\section{REFERENCES}

[1] Williams M.L.: Stress singularities resulting from various boundary conditions in angular corners of plates in extension, J. Appl. Mech., Vol. 74, pp. 526-528 (1952).

[2] Williams M.L.: The stress around a fault or crack in dissimilar media, Bull. Seism. Soc. of America, Vol. 49, pp. 199-204 (1959).

[3] Bogy D.B.: Edge-bonded dissimilar orthogonal elastic wedges under normal and shear loading, J. Appl. Mech., Vol. 25, pp. 460-466 (1968).

[4] Bogy D.B.: Two edge-bonded elastic wedges of different materials and wedge angles under surface tractions, J. Appl. Mech., Vol. 38, pp. 377-386 (1971).

[5] Theocaris P.S.: The order of singularity at a multi-wedge corner of a composite plate", Int. J. Engng. Sci., Vol. 12, pp. 107-120 (1974).

[6] Bogy D.B., Wang K.C.: Stress singularities at interface corners in bonded dissimilar isotropic elastic materials, Int. J. Sol. Struct., Vol. 7, pp. 993-1005 (1971).

[7] Hein V.L., Erdogan F.: Stress singularities in a two-material wedge, Int. J. Fract. Mech., Vol. 7, pp. 317-330 (1971).

[8] Chen D., Nisitani H.: Singular stress field near the corner of jointed dissimilar materials, $J$. Appl. Mech., Vol. 60, pp. 607-613 (1993).

[9] Pageau S.S., Joseph P.F., Biggers S.B. Jr.: The order of stress singularities for bonded and disbonded three-material junctions, Int. J. Sol. Struct., Vol. 31, 2979-2997 (1994).

[10] Carpinteri A., Paggi M.: Interface crack propagation in concrete composites: asymptotic analysis of stress-singularities, Proceedings of the FraMCoS-5 Post-Conference Workshop on Interface Modeling, Vail, Colorado, 20-23 (2004).

[11] Carpinteri A.: Stress-singularity and generalized fracture toughness at the vertex of reentreant corners, Engng. Fract. Mech., Vol. 26, pp. 143-155 (1987). 